Book Review

\title{
Han Yu (2015). The Other Kind of Funnies: Comics in Technical Communication. New York, NY: Baywood Publishing Company.
}

Reviewed by Suzanne Rintoul

Conestoga College, Canada

Han Yu's The Other Kind of Funnies: Comics in Technical Communication challenges the notion that technical writing is too "rational" or "serious" to accommodate the conventions of comicsstyle communication. She does this by illustrating comics' unique ability to distill and reinforce information in ways entirely appropriate not just for complementing the purposes of many technical writers, but also for fulfilling the needs of their diverse audiences. The book's major strength lies in Yu's capacity to locate the productive nexus between two ostensibly dissimilar modes so that by the final chapter those connections seem not only probable, but natural. This text will be especially useful to scholars of rhetoric (particularly those invested in visual culture and/or technical writing) and practitioners of technical writing eager to embrace new (or in some cases re-embrace older) ways of seeing the relationship between textual and visual elements. The clarity with which $\mathrm{Yu}$ distils complex theoretical concepts makes this book appropriate reading for undergraduate or graduate courses as well as for non-scholarly audiences.

The book is divided into two parts: first, a historical background of both comics and technical communication; and second, a comprehensive study of multiple genres of what Yu refers to as “comics-style technical communication." While intersecting histories of comics and technical communication to illustrate their convergences and potential, The Other Kind of Funnies uses a cross-cultural approach - focusing largely on Japanese, French, Latin American and US texts. This approach reveals how comics appeal to a diverse audience while avoiding the pitfalls of alienating non-native speakers like text-based approaches have done in the past. Comics-style technical communication, Yu convincingly argues, encourages reader engagement through the construction of enjoyable user experiences, defamiliarization, participatory reading, opportunities for reader-identification, and, in cases where technical communication must convince readers, covert and thus non-threatening forms of persuasion.

$\mathrm{Yu}$ does not, however, dismiss the limitations of integrating comics into technical communication, though she is careful to avoid reading their perceived lack of technicality as a 
significant problem. Yu underscores the importance of the writing situation to the usefulness of comics and adeptly identifies contexts in which comics are simply not the appropriate medium for an author's purpose. Comics are best suited, she argues, for narrative-based forms of communication that benefit from the portrayal of users and objects, and for "absolute" contexts that encourage readers to consider only one response or action. And even in these situations, $\mathrm{Yu}$ notes, the imagery used in comics can create challenges related to the cultural specificity of visual references, their almost inevitably affective elements, and their perceived nonseriousness. The book is therefore not suggesting that comics belong everywhere that technical information does.

The book's section on genre studies - including chapters on instructional comics, development comics, educational comics, and propaganda comics - shows just where comics do work. Each chapter in this section is divided into two parts: examples of a given genre, and techniques that render these examples effective in technical communication contexts. In keeping with the crosscultural approach, Yu draws not just on genres of technical writing that would be familiar to a North American audience, but also on Japanese, Latin, and French traditions. "Instructional Comics," for instance, discusses examples of safety comics and military comics as well as work manga - bildungsroman-style Japanese narratives intended to instruct young boys, primarily, how to execute a number of tasks through hard work and integrity. Yu claims that techniques in instructional comics are suitable for technical communication for a variety of reasons: narratives themselves help readers to make sense of their lives and worlds; surrogate users in such narratives demonstrate skills and procedures; frequently anthropomorphized tools or parts encourage readers to empathize with whatever mechanism they may be struggling with; and, generally speaking, comics-style instructions provide emotional relief and reassurance, which is particularly helpful when reading emergency material.

Building on these ideas, Yu notes that development and educational comics likewise aid readers in gleaning and even creating meaning from technical documents. "Development Comics" makes the claim that comics-style communication is ideal for social justice and activist texts insofar as it has a strong capacity for reaching the audiences such documents are intended to help, and thus for achieving targeted outcomes for particular groups. For example, superhero comics (more popular in North American) and fotonovelas (more popular among Latino audiences) draw on analogies, narrative, and visual appeal to engage readers in developmental campaigns on topics ranging from safer sex to workplace guidelines for dealing with harassment. The chapter that follows makes an important distinction between development and educational comics, noting that while developmental work is typically intended for the less privileged reader, educational 
comics target those readers immersed in discipline-specific study. Illustrating what she refers to as the Japanese belief that comics can teach, Yu focuses largely on benkyo manga, a form of educational comic used at all levels of Japanese education, but she also notes that comics are indeed often also used in the US to teach historical narratives and to render classic literature more accessible to readers. The multimodal design of comics-style educational material, Yu notes, stimulates both sides of the brain and helps learners with both design and pattern recognition. And, she reminds us, the affective responses elicited by comics can make curriculum more fun, which facilitates learning generally.

Closing this section is a chapter on propaganda comics, in which Yu returns to perhaps the most interesting thread in her book: comics-style technical communication has a unique capacity for persuasion. Drawing on well and lesser-known examples of governmental uses of this genre in American and Japan, Yu identifies particular characteristics of propaganda comics that allow them to change people's mindsets in unthreatening ways. Exaggeration, for instance, allows images to take on more meanings, alternately promoting positive or negative traits of individuals and groups, while model characters allow audiences to observe and recreate ideal behaviours. The narrative style of comics, meanwhile, ensures that the persuasive process remains gradual and therefore more effective for skeptical readers.

The Other Kind of Funnies ends with a chapter that asks simply, "Where Do We Go From Here?" To respond, Yu stresses the importance of embracing comics-style technical communication in spite of comics' reputation as a "laypersons' medium" (p. 250) because to do so would better serve readers and the profession generally. She acknowledges the various challenges that such a shift in the field would bring, particularly in terms of production and training, and in turn makes a strong case for proactive changes to methods of the teaching and academic study of technical communication. Overall, this book offers a significant contribution to the field insofar as it proposes major paradigm shifts for scholars, teachers, and practitioners, combatting prejudice against comics to produce tangible benefits for audiences. 